\title{
Charged bosons in a quasi-one-dimensional system
}

\author{
B. Tanatar, ${ }^{1}$ Bahman Davoudi, ${ }^{2}$ and Mohammad Kohandel ${ }^{2,3}$ \\ ${ }^{1}$ Department of Physics, Bilkent University, Bilkent, Ankara 06533, Turkey \\ ${ }^{2}$ Institute for Studies in Theoretical Physics and Mathematics, Tehran 19395-5531, Iran \\ ${ }^{3}$ Department of Physics, Shahid Rajaee University, Tehran, Iran
}

(Received 1 May 2000)

\begin{abstract}
The ground-state properties of a system of charged bosons in a quasi-one-dimensional model with a neutralizing background are investigated within the hypernetted-chain approximation. Strong correlation effects drive the system from a homogeneous fluid phase toward a more ordered structure akin to Wigner crystallization in higher-dimensional charged quantum systems. The ordered phase of charged bosons is signaled by the development of a peak in the static structure factor, which is analyzed as a function of the density and the lateral width of the one-dimensional structure. We also calculate the pair-distribution function, the ground-state energy, and the local-field correction, and compare our results with other theoretical approaches.
\end{abstract}

\section{INTRODUCTION}

Models of a quasi-one-dimensional (Q1D) electron system, in which the electrons are free to move in only one spatial direction, are useful to understand the properties of semiconductor quantum wires and naturally occurring organic materials. ${ }^{1}$ As a many-body problem, electrons interacting via long-range Coulomb potential offers a suitable testing ground to model more realistic systems mentioned above and to compare with exact results obtained for a variety of model problems. ${ }^{2}$ The closely related system of charged bosons (charged particles obeying Bose-Einstein statistics) subject to the same confinement conditions is also of recent interest. ${ }^{3-5}$ Part of the motivation comes from the recent excitement over Bose-Einstein condensed atomic systems. From a theoretical point of view, by comparing the results for charged Bose systems with those for the electron gas one may assess the role of correlation effects in reduced dimensions. Moreover, the possibility of realizing a charged boson system in semiconductor heterostructures was also suggested. ${ }^{6}$

In the case of quasi-one-dimensional electron systems, the correlation effects drive the system to an ordered phase as signaled by the presence of a peak in the ground-state structure factor. ${ }^{7}$ This observation was subsequently put on firmer ground through the recent quantum Monte Carlo (QMC) simulations. $^{8}$ The picture that emerges from various theoretical approaches vis à vis the QMC results is that, even though perturbation theory or some other integral-equationbased approximate schemes may be reliable in getting accurate ground-state energies, they differ drastically in their predictions of the correlation functions. This fact has been pointed out by Malatesta and Senatore ${ }^{8}$ as a test to assess the quality of approximate theories against the QMC results.

In this work, we employ the hypernetted-chain (HNC) approximation as applied to homogeneous quantum liquids to calculate the ground-state correlation functions in a model quasi-one-dimensional system. ${ }^{9}$ Our aim is to demonstrate the significantly different behavior of the static structure factor and pair-distribution function at low density, compared to the uniform limit and some other theoretical approaches. The peak structure emerging in the correlation functions may be considered as signaling a transition to an ordered phase. Similar observations were made in a Q1D electron system (quantum wire) within a theory that takes dynamical correlations into account. ${ }^{7}$ Thus, the accurate treatment of correlation effects in charged quantum fluids (irrespective of their statistics) is essential in detecting the phase transitions. The variational approach to the many-boson problem has recently been applied to a number of neutral bosonic systems with great success. ${ }^{10}$

In the following, we first introduce the model of a Q1D charged boson system, and outline the calculational framework of the HNC approximation. We then present our results for the static correlation functions and ground-state energy and compare them with other calculations. We conclude with a brief discussion on further work and a summary.

\section{MODEL AND THEORY}

Similarly to the quantum wire models of an electron gas, we consider a charged boson fluid in $1 \mathrm{D}$ embedded in a rigid neutralizing background. The system is characterized by the density parameter $r_{s}$ defined in terms of the linear particle density $\rho$ and effective Bohr radius $a_{B}^{*}$, through $r_{s}=1 /\left(2 \rho a_{B}^{*}\right)$. A parabolic confinement potential gives the charged bosons free motion in one spatial direction, say the $z$ direction and a finite extent in the transverse directions which may be characterized by a width parameter $b$. Using the specific model of harmonic confinement first developed by Friesen and Bergersen, ${ }^{11}$ the effective Coulomb potential in configuration space can be written as

$$
V(z)=\frac{\sqrt{\pi}}{2 b} \exp \left[\frac{z^{2}}{4 b^{2}}\right] \operatorname{erfc}\left[\frac{|z|}{2 b}\right],
$$

which yields

$$
V(q)=E_{1}\left(q^{2} b^{2}\right) \exp \left(q^{2} b^{2}\right),
$$

in Fourier space, where $E_{1}(x)=\int_{x}^{\infty} d t e^{-t} / t$ is the exponential integral. The above form of the effective interaction exhibits typical $1 \mathrm{D}$ behavior $V(q) \sim \ln (q b)$ as $q \rightarrow 0$ and 3D 
behavior $V(q) \sim 1 /(q b)^{2}$ as $q \rightarrow \infty$. The very same confinement model has been used for electron gas ${ }^{11,12}$ and charged boson ${ }^{4}$ problems in different approximation schemes.

We calculate the ground-state energy and structure of the charged boson system within a variational many-body theory, using the potential of interaction between the charged bosons as input. Minimization of the energy expectation value, and using the hypernetted-chain equation to relate the distribution function to the two-body correlation function leads to the so-called $\mathrm{HNC} / 0$ approximation (we use $\mathrm{HNC}$ for short) when the elementary diagrams are neglected. The details of the derivation in one-dimensional systems are given by Krotscheck, Miller, and Wojdylo ${ }^{10}$ and a general discussion of the variational approach can be found in the review by Krotscheck and Saarela. ${ }^{13}$ In this approach the static structure factor is expressed as

$$
S(q)=\left[1+\frac{4 m}{q^{2}} V_{p-h}(q)\right]^{-1},
$$

(we take $\hbar=1$ ) in terms of the particle-hole interaction $V_{p-h}(q)$, which written in the real-space reads

$$
V_{p-h}(z)=g(z) V(z)+\frac{1}{m}\left|\frac{d}{d z} \sqrt{g(z)}\right|^{2}+[g(z)-1] w_{\text {ind }}(z) .
$$

Here $w_{\text {ind }}(z)$ is the induced interaction that needs to be calculated self-consistently from the Fourier transform

$$
w_{\text {ind }}(q)=-\frac{q^{2}}{2 m}[S(q)-1]-V_{p-h}(q) .
$$

Once the $S(q)$ and $g(z)$ are determined within the selfconsistent HNC equations, the ground state energy (per particle) can be evaluated from ${ }^{10}$

$$
\begin{aligned}
\frac{E}{N}= & -\frac{1}{8 m \rho} \int_{-\infty}^{\infty} \frac{d q}{2 \pi} q^{2} \frac{[S(q)-1]^{3}}{S(q)} \\
& +\frac{\rho}{2} \int_{-\infty}^{\infty} d z\left[[g(z)-1] V(z)+\frac{1}{m}\left|\frac{d}{d z} \sqrt{g(z)}\right|^{2}\right],
\end{aligned}
$$

where the first and second terms may be identified as the kinetic and potential energies (per particle), respectively. Other thermodynamic quantities are calculated from the ground-state energy, i.e., pressure $P / \rho=\rho(d / d \rho)(E / N)$ and chemical potential $\mu=E / N+P / \rho$. The compressibility is given by $1 /(\rho \kappa)=\rho d \mu / d \rho$. As noted by Krotscheck, Miller, and Wojdylo ${ }^{10}$ the above formulation of the HNC theory is adequately optimized for one-dimensional systems. Thus, in contrast to the Singwi-Tosi-Lnad- and Sjölander (STLS)-theory ${ }^{14}$-based works before, ${ }^{4,5}$ we do not assume the existence of a condensate.

In the above expressions, if we replace the selfconsistently calculated particle-hole interaction $V_{p-h}(q)$ by the bare effective Coulomb potential $V(q)$, the resulting $S(q), g(z), E / N$, and the rest of the thermodynamic quantities constitute the uniform limit $^{7}$ [or the random-phase approximation (RPA)].

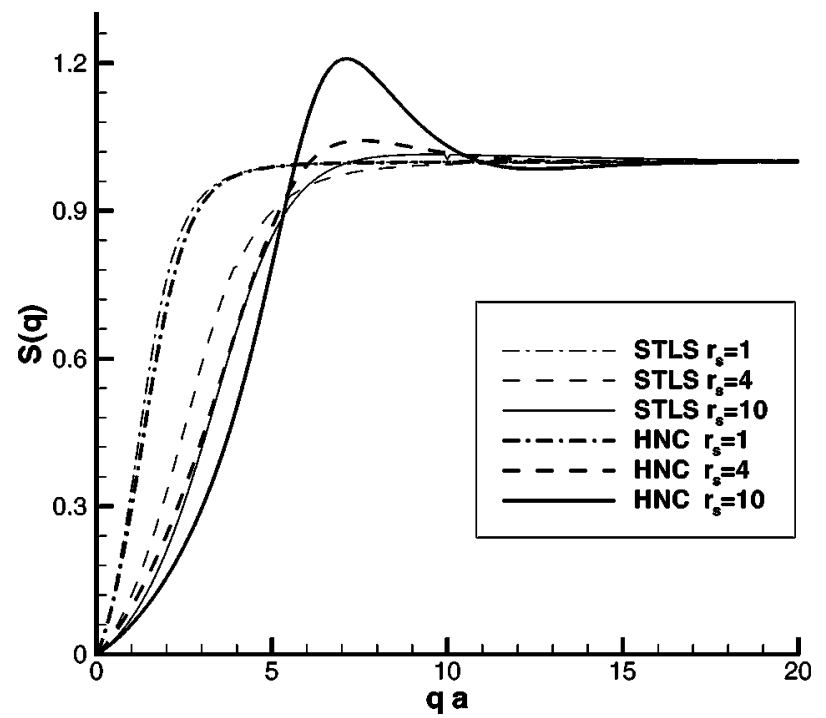

FIG. 1. The static structure factor $S(q)$ for 1D charged bosons of lateral width $b=a_{B}^{*}$ at various $r_{s}$ values. The thick and thin lines correspond to the results of HNC and STLS (from Ref. 4) approximations, respectively.

\section{RESULTS AND DISCUSSION}

In this section we present the results of the numerical calculations for the ground-state correlation functions and energy. We solve the set of self-consistent equations within the HNC approximation with the potential defined in Eq. (1) as input, and find the static structure factor, the pair correlation function, and the effective particle-hole interaction $V_{p-h}(q)$, for various densities characterized by $r_{s}$ and width parameter $b$. In the numerical calculations we use the following scaled variables: the average interparticle distance $a$ $=1 /(2 \rho)$ for length and the effective Rydberg constant $R^{*}$ $=1 /\left(2 m a_{B}^{* 2}\right)$ for energy.

We first display in Fig. 1 the static structure factor $S(q)$ for Q1D charged bosons for various values of the density parameter $r_{s}$, where the width parameter is $b=a_{B}^{*}$. We find that as the density is decreased the correlation effects become stronger and the $S(q)$ starts to develop a broad peak around $q a \simeq 6.5$. In the same figure we also plot for comparison the results of the static STLS calculation performed for the same confinement model and parameters. In the static STLS approach, the correlation effects are taken into account by a local-field correction factor calculated self-consistently. ${ }^{14}$ The static STLS results of Gold and Calmels ${ }^{4}$ do not show any peak as the density decreases. Similar qualitative results are obtained in a different model ${ }^{4}$ for Q1D charged bosons within the STLS approximation that does not yield any peaked structure factor. The general behavior of $S(q)$ in STLS-type calculations ${ }^{4,5}$ is somewhat similar to the random-phase approximation (RPA) results. The uniform limit or the RPA of the present model has been discussed previously. ${ }^{4,5}$ The RPA overestimates the correlation effects and breaks down as $r_{s} \gtrsim 2$. The STLS approximation yields accurate ground-state energies but the resulting $S(q)$ does not show a peak, as compared to the HNC approximation. With increasing lateral width $b$, the onset of the peak structure in $S(q)$ increases to higher values of $r_{s}$.

The Fourier transform of $S(q)$ yields the pair-distribution 


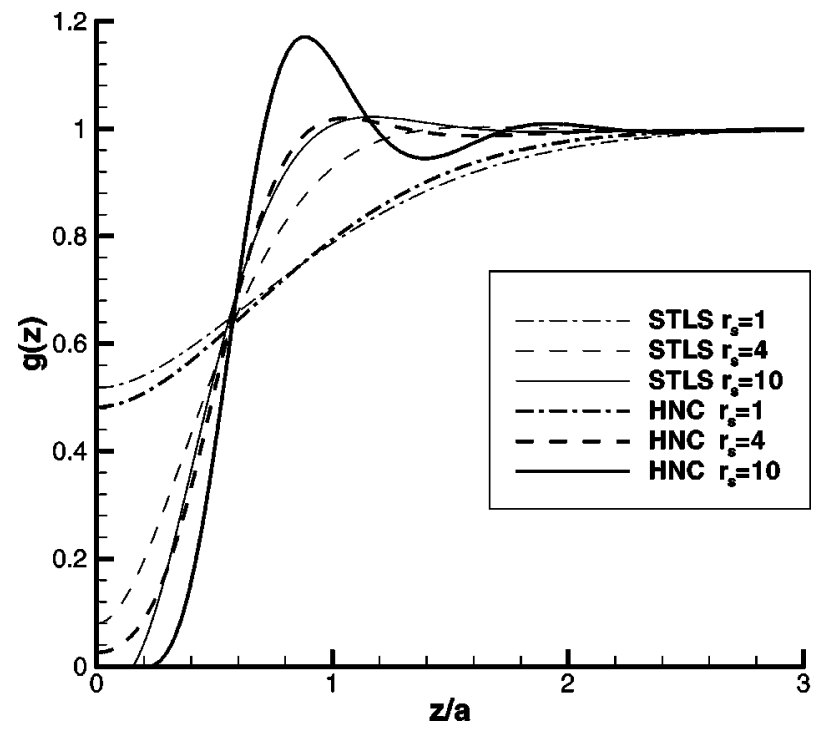

FIG. 2. The pair-distribution function $g(z)$ for $1 \mathrm{D}$ charged bosons of lateral width $b=a_{B}^{*}$ at various $r_{s}$ values. The thick and thin lines correspond to the results of HNC and STLS (from Ref. 4) approximations, respectively.

function $g(z)$, namely, the probability of finding a boson at some distance $z$, if another one is placed at the origin. It is well known that the oscillatory behavior of the $g(z)$ is a signature of an ordered phase; therefore, the system is going to have a transition from the liquid phase to the solidlike phase with decreasing density. Since there is no true longrange order in one-dimensional systems, the new phase should have quasicrystalline character. The properties of this partially ordered state and its more accurate dependence on the density and width parameter needs to be further explored. For instance, QMC simulations of the ordered phase and density functional theory of freezing may be employed to determine the transition density. Figure 2 shows the gradual development of a peak in $g(z)$ as $r_{s}$ increases. At the same time, the value of $g(0)$ decreases with increasing $r_{s}$, but remains positive for a large range of $r_{s}$, consistent with the probabilistic definition. The $g(0)$ usually becomes negative as $r_{s}$ increases in other approximate theories, making the correlation functions somewhat unreliable. We find similar emergence of a peak in the static structure factor and enhanced oscillations in the pair-distribution function for other values of the width parameter $b$.

The ground-state energy as a function of the density parameter $r_{s}$, for two values of the width parameter $b$, is shown in Fig. 3. Also shown in the same figure are the ground-state energies calculated within the sum-rule approach of the STLS theory. ${ }^{4}$ We find that the agreement in $E\left(r_{s}\right)$ in both approaches is very good, and surmise that similar level of agreement can be attained in QMC calculations, as was the case in Q1D electron systems ${ }^{7}$ and in higher dimensional charged boson systems. ${ }^{3,15}$ The uniform limit or the RPA results (not shown here) already exhibit departure from those of HNC and STLS approximations starting from $r_{s} \gtrsim 2$, which has been noted in the previous calculations. ${ }^{4,5}$ The results on the ground-state energy imply that $E\left(r_{s}\right)$ does not depend on the actual structure in $S(q)$ or $g(z)$ very critically, although the correlation functions may have quite different behavior indicating possible phase transitions.

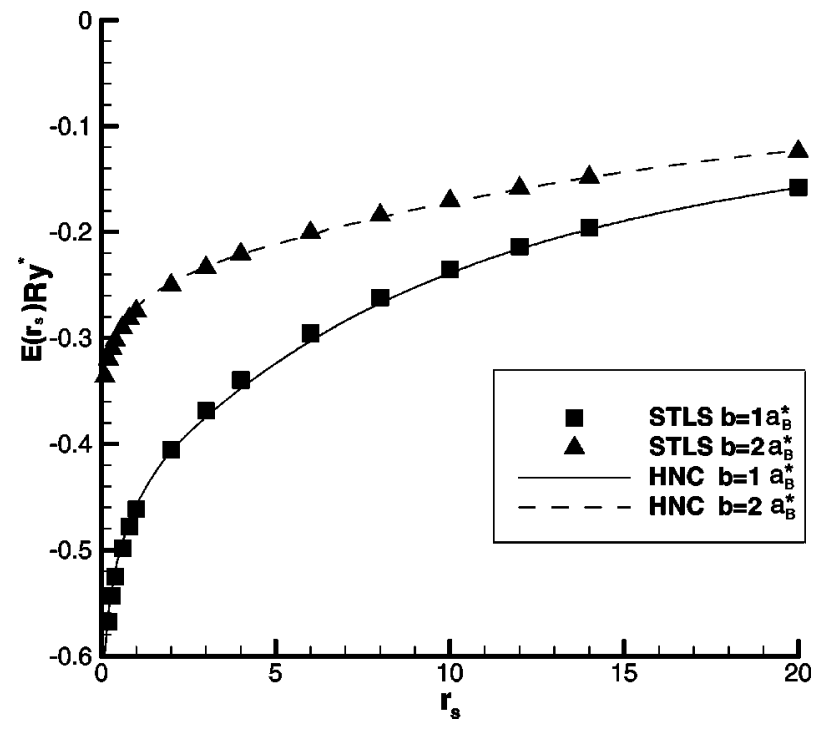

FIG. 3. The ground-state energy $E\left(r_{s}\right)$ per particle of Q1D charged bosons within the HNC and STLS approximations as a function of $r_{s}$ for two values of the width parameter. The points referring to the STLS calculation are taken from Ref. 4.

The correlation effects beyond the random-phase approximation are usually described by the local-field correction defined as $V_{\text {eff }}(q)=V(q)[1-G(q)]$, by rewriting the effective many-body interaction. If we identify our $V_{p-h}(q)$ with the effective interaction we can deduce the corresponding localfield correction within the HNC approximation. The Bogoliubov relation $\left[\mathrm{Eq}\right.$. (3)] provides the expression $G_{\mathrm{HNC}}(q)$ $=1-\left(q^{2} / 4 m\right)\left[1 / S^{2}(q)-1\right] / V(q)$. In Fig. 4 we show $G_{\mathrm{HNC}}(q)$ and the local-field correction obtained in the static STLS approximation $G_{\text {STLS }}(q)$ as a function of $q$ for three values of the density parameter. The $G_{\mathrm{HNC}}(q)$ typically exhibits a peak at intermediate to high values of $q$, whereas the $G_{\mathrm{STLS}}(q)$ is a monotonic function similar to the electron gas case. ${ }^{12,7}$ Figure 4 also indicates that the very large $q$ limit of the respective local-field corrections in the HNC and STLS approximations is quite different, which elicits further inves-

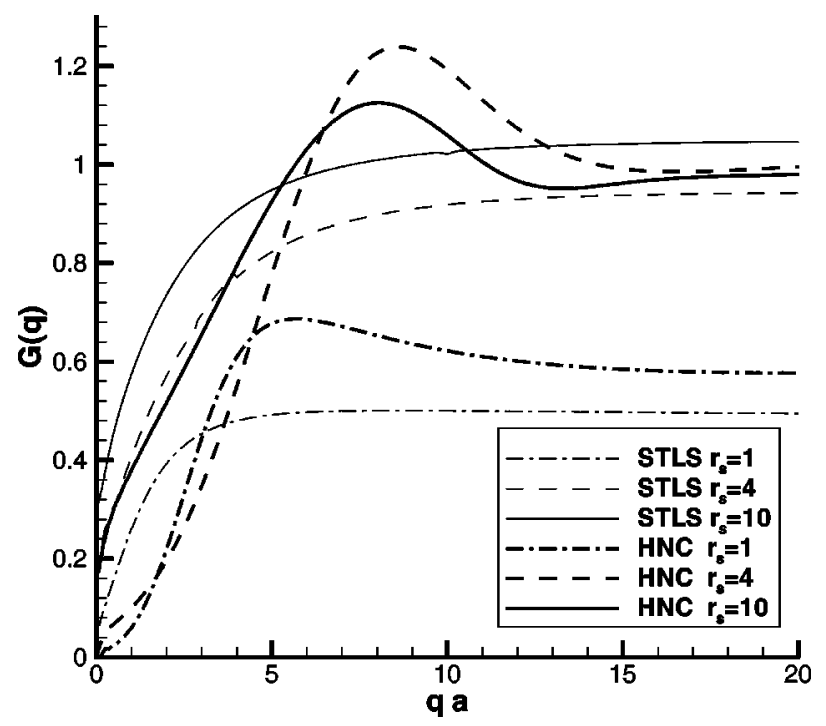

FIG. 4. The local-field correction factor $G(q)$ extracted from the HNC scheme in comparison to the STLS approximation. 
tigation in terms of sum rules. ${ }^{3}$

There are several other physical properties of charged bosons in 1D that we have not addressed in this short paper. The dielectric properties to be obtained from a suitably defined dielectric function $\varepsilon(q, \omega)$ would give information on the collective charge excitations in the system in terms of their dispersion and damping behavior. The higher-order corrections such as elementary diagrams thought to be not important for ground-state energy may have important contributions to the dynamical properties. Work in these directions is in progress.

In summary, we have calculated the ground-state energy and correlation functions in a quasi-one-dimensional charged Bose fluid within the HNC approximation. The calculated correlation functions signal the development of an ordered phase at low density similarly to the situation in electron gas systems. It would be interesting to perform QMC simulations for this model system to test and verify some of our predictions and to calculate the transition density.

\section{ACKNOWLEDGMENTS}

The work of B.T. is partially supported by the Scientific and Technical Research Council of Turkey (TUBITAK) under Grant No. TBAG-1162 and NATO under Grant No. SfP971970. B. D. and M. K. acknowledge support from the Institute for Studies in Theoretical Physics and Mathematics, Tehran, Iran. We thank Professor G. Senatore for sending us a copy of A. Malatesta's thesis.
${ }^{1}$ See for instance, N. H. March and M. P. Tosi, Adv. Phys. 44, 299 (1995); C. Weisbuch and B. Vinter, Quantum Semiconductor Structures (Academic, Boston, 1991).

${ }^{2}$ D. C. Mattis, The Many-Body Problem, An Encyclopedia of Exactly Solved Models in One Dimension (World Scientific, Singapore, 1993).

${ }^{3}$ V. Apaja, J. Halinen, V. Halonen, E. Krotscheck, and M. Saarela, Phys. Rev. B 55, 12925 (1997); and references therein.

${ }^{4}$ A. Gold and L. Calmels, Phys. Rev. B 57, 12119 (1998); L. Calmels and A. Gold, J. Phys. IV 347 (2000).

${ }^{5}$ R. K. Moudgil, K. Tankeshwar, and K. N. Pathak, J. Phys.: Condensed Matter 11, 4665 (1999).

${ }^{6}$ L. D. Shvartsman and J. E. Golub, in Bose-Einstein Condensation, edited by A. Griffin, D. W. Snoke, and S. Stringari (Cambridge, New York, 1995), p. 552.

${ }^{7}$ C. Bulutay and B. Tanatar, Europhys. Lett. 43, 572 (1998); B.
Tanatar and C. Bulutay, Phys. Rev. B 59, 15019 (1999).

${ }^{8}$ A. Malatesta and G. Senatore, J. Phys. IV 10, 341 (2000); A. Malatesta, Ph.D. thesis, University of Trieste (1999).

${ }^{9}$ E. Feenberg, Theory of Quantum Liquids (Academic, New York, 1969).

${ }^{10}$ E. Krotscheck, M. D. Miller, and J. Wojdylo, Phys. Rev. B 60, 13 028 (1999); E. Krotscheck and M. D. Miller, ibid. 60, 13038 (1999).

${ }^{11}$ W. I. Friesen and B. Bergersen, J. Phys. C 13, 6627 (1980).

${ }^{12}$ L. Calmels and A. Gold, Phys. Rev. B 56, 1762 (1997).

${ }^{13}$ E. Krotscheck and M. Saarela, Phys. Rep. 232, 1 (1993).

${ }^{14}$ K. S. Singwi, M. P. Tosi, R. H. Land, and A. Sjölander, Phys. Rev. 176, 589 (1968); K. S. Singwi and M. P. Tosi, Solid State Phys. 36, 177 (1981).

${ }^{15}$ S. Moroni, S. Conti, and M. P. Tosi, Phys. Rev. B 53, 9688 (1996). 